\title{
Analysis of Queuing Scheduling Linkage Model to Minimize the Hiring Cost of Machines/Equipments
}

\author{
Sameer Sharma $^{a}$, Deepak Gupta ${ }^{b}$, Seema Sharma \\ ${ }^{a}$ Department of Mathematics, D.A.V. College, Jalandhar, Punjab, India \\ ${ }^{b}$ Department of Mathematics, M.M. University, Mullana, Haryana, India \\ Email: samsharma31@gmail.com
}

\begin{abstract}
Hiring of machines/equipment is growing as the most preferred alternative method for financing the latest technology in many developing countries due to budgetary constraints faced by most of industries. The present paper is an attempt to develop an algorithm to minimize the utilization time of machines/equipments taken on rent and hence their hiring cost for the proposed queuing scheduling linkage model. The phase I of service (network of queues) consists of parallel and biserial servers in which the arrival and the service pattern both follows poisson's law. Further, the completion time of jobs in getting phase I of service will be the setup time for first machine in phase II of service consisting of ' $m$ ' machines/equipments in series taken on rent. In industries men, machines, materials and money are involved for production. The manager of an industry is interested to use them in an economic manner so that the cost associated with the production is not increased or it minimum in the competition market. In industry there are some very complicated machines that are often necessary for performing a variety of procedures. In order to stay competitive, up to date equipments are required and buying all of these equipments get really expensive over time. There are industries who take various machines/equipment on rent and hiring of equipment is an affordable and quick solution for many industries, which are presently constrained by the availability of limited funds due to the recent global economic recession. Renting of equipments/machines enable saving working capital, give options for upgradation to new technology. The objective of paper is to find the latest time at which machines/ equipments should be hired so as to minimize utilization time and hence their hiring cost with minimum total elapsed time and with minimum mean queue length for the proposed queuing scheduling linkage model. A numerical illustration is also carried to test the efficiency of the proposed algorithm.
\end{abstract}

Keywords: Flowshop scheduling, Mean queue length, Utilization time, Waiting time, Hiring Cost 


\section{INTRODUCTION}

Waiting lines or queues are a common occurrence in everyday life and in many industrial situations. We all observe queues in communication systems, voice or data traffic queues for the transmission, in manufacturing system with several work stations, units completing work in one station wait for access to the next, patients arriving at hospital or a clinic for treatment etc. The amount of time that a nation's population wastes by waiting in queues is a major factor in both the quality of life there and the efficiency of the nation's economy. Queuing theory uses mathematical tools to predict the behaviour of queuing system consisting of a stream of arriving customers, a queue and a service stage. Prediction deals with mean length of queue, mean waiting time, total completion time and so on. Also, the scheduling models concerned with determination of an optimal sequence in which various jobs/customers are served in order to minimize the total elapsed time and some other measure of performance. Scheduling problems exist whenever there is an alternative choice in which a number of jobs/tasks can be performed. In this paper the processing of jobs/customers through a linkage network of queues consisting of biserial and parallel servers linked to a common server with a flowshop scheduling system having ' $m$ ' machines/equipments in series taken on rent is considered. The practical situations of the proposed work can be taken as examples in medical industry, construction industry and manufacturing industry etc. In hospitals, the patients arriving have to be passed through a series of servers / channels / stages before being attended by a doctor/ expert and there after they have to be processed through a number of hired equipments/machines in series. Because of growing tendency for emergency cases, the hospitals have been experiencing a counting increase in the number of patient visits every day. As a result, it has become quite common for the patients arriving during peak usage hour have to wait until it is there turn to be treated by the doctor. Also, the medical science can save the patient's life but proper care leads to a faster recovery. Care giving techniques often require hi-tech up-to date medical equipments and most of these equipment's are expensive. Further, they are often needed for a few days or weeks thus buying them do not make much sense even if one can afford them. Rental of medical equipment/machine is an affordable and quick solution for hospitals, nursing homes, physicians, which are presently constrained by the availability of limited funds. Global Industry Analyst (GIA) announces that the global market for medical equipment rental and leasing is forecast to reach $\$ 56$ billion by the year 2017. Major factor propelling market growth include growing incidence of chronic diseases, the demand for diagnosis, technological advancements, leading to the obsolescence of old equipments, surging prices of medical equipments and need to curb healthcare expenses. As another example, in construction industry rental of equipment offers contractors and construction companies a cost effective alternative to owing equipments because equipments become a fixed cost without the accompany overhead of ownership such as maintenance, repair and storage. The rental company handles and pays for the warranty, maintenance and up keep the equipment. The worldwide construction equipment rental market is projected to reach an estimated \$193 billion by 2017.

The rest of paper is organized as follows: Section 2 deals with review of existing literature. Section 3 introduces the mathematical model and analysis of proposed linkage model with some basic definitions, various symbols and fundamental theorems required in the progress of the paper. Section 4 describes the algorithm proposed to optimize various characteristics of the proposed model. Section 5 presents a numerical illustration to test the efficiency of the algorithm proposed. The paper is concluded in section 6 followed by the references.

\section{LITERATURE REVIEW}

The origin of queuing theory can be tracked back to early in the last century when Erlang (1909), a Danish engineer, applied this theory extensively to study the behavior of telephone networks. Jackson (1954) studied the behaviour of a queuing system containing phase type service. Little (1965) gave the formula for calculating the mean queue length. Maggu (1970) introduced the concept of bitendom in theory of queues. Later on various ideas were developed by many researchers with different modifications and argumentations. Singh et al. (2005) studied the transient behaviour of a queuing network with parallel bi-series queue linked with a common channel. Gupta et al. (2011) studied steady state behaviour of a queue model with biserial servers and parallel servers linked in series with a common server. Also, one of the earliest results in flow shop scheduling theory is an algorithm by Johnson's (1954). Smith (1956) whose work is one of the earliest considered minimization of mean flowtime and maximum tardiness. Bagga (1968) studied the sequencing of jobs in flowshop scheduling when the machines are taken on rent. Bagga and Bhambani (2000) obtained an optimal sequence for minimizing the bicriteria for the general $n \times m$ flowshop problem. Sharma and Gupta (2011) minimized the rental cost under a specified rental policy in two stage flowshop. Gupta, Sharma and Gulati (2011) studied the bicriteria in three stage flowshop scheduling to minimize the rental cost of machines with minimum makespan. 
The literature reveled that a lot of research work has already been done in the field of Queuing and Scheduling theory individually. Only some efforts have been made to establish a linkage between these two fields of optimization. Singh and Kumar (2009), Maggu and Gupta (2007), Gupta et al. (2012a, 2012b, 2012c, 2012d) have made some efforts to optimized the total flow time, waiting time and service time in Queuing-Scheduling linkage model. Recently Gupta et al. (2013) have established a linkage model of network of queues with flow shop scheduling system including independent transportation time. This paper establishes link between network of queues given by Gupta et al. (2011) with a flowshop scheduling having a system of equipments/machines taken on rent to minimize their hiring cost with minimum makespan as given by Sharma and Gupta (2012d).

\section{MATHEMATICAL MODEL AND ANAYSIS}

The entire queue model is comprised of three service servers $S_{1}, S_{2}$ and $S_{3}$. The server $S_{1}$ consists of two

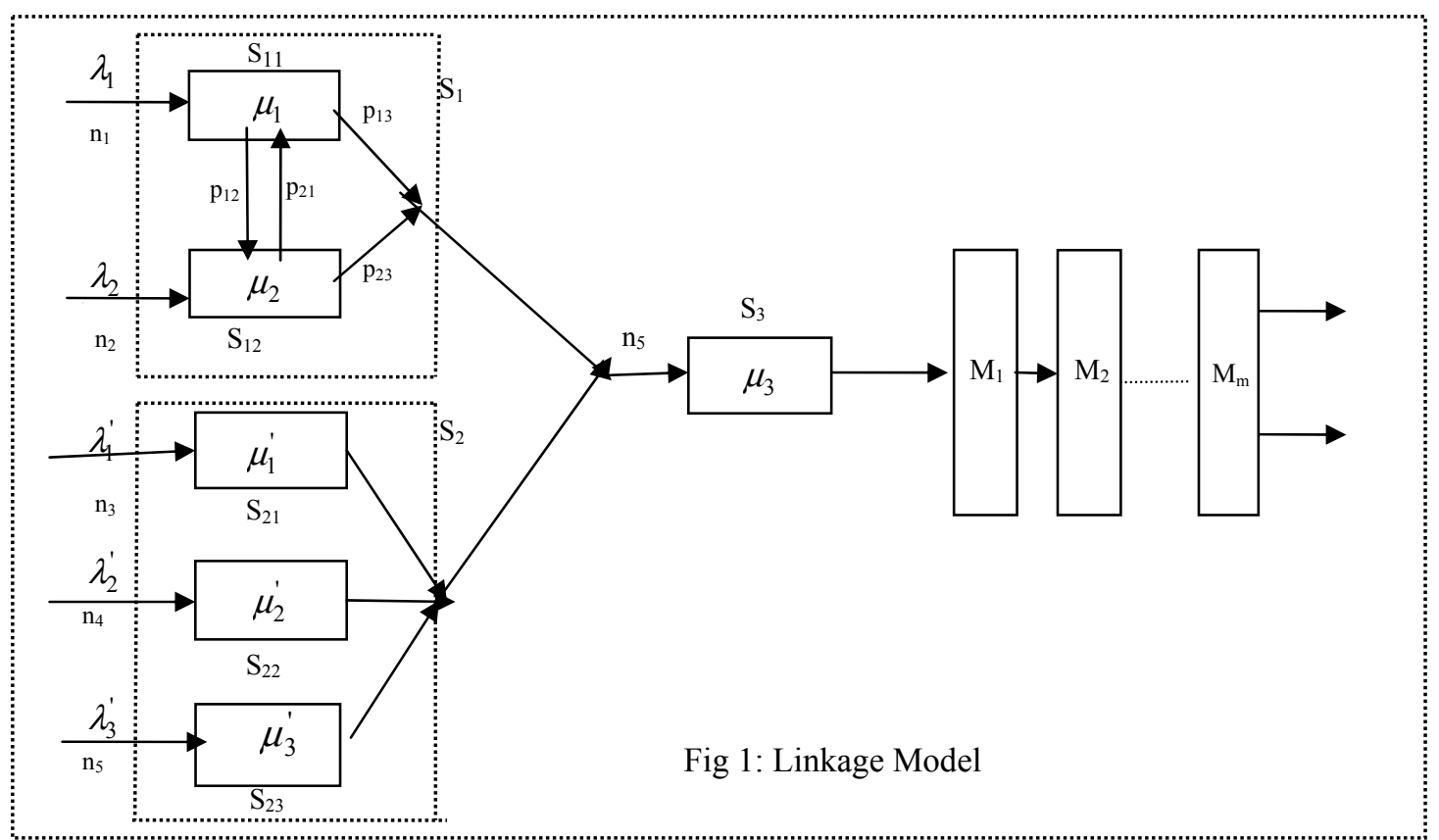

biserial service sub-servers $S_{11}$ and $S_{12}$. The server $S_{2}$ contains three parallel sub-servers $S_{21}, S_{22}$ and $S_{23}$. The service server $S_{3}$ is commonly linked in series with each of two servers $S_{1}$ and $S_{2}$ for competition of the phase I of service demanded either at the server $S_{1}$ or $S_{2}$. The service time at the servers $S_{11}, S_{12}, S_{21}, S_{22}, S_{23}$ and $S_{3}$ are distributed exponentially with mean service rate $\mu_{1}, \mu_{2}, \mu_{1}^{\prime}, \mu_{2}^{\prime}, \mu_{3}^{\prime}$ and $\mu_{3}$ respectively. Queues are said to be formed in front of the service servers if they are busy. Customers arriving at the rate $\lambda_{1}$ after completion of phase service at $S_{11}$ will join server $S_{12}$ or $S_{3}$.i.e. either processed through the network of servers $S_{11} \rightarrow S_{12} \rightarrow S_{3}$ or $S_{11} \rightarrow S_{3}$ with the probabilities $p_{12}$ or $p_{13}$ such that $p_{12}+p_{13}=1$ and those arriving at the rate $\lambda_{2}$ after completion of phase service at $S_{12}$ will join sever $S_{11}$ or $S_{3}$.i.e. either processed through the network of servers $S_{21} \rightarrow S_{11} \rightarrow S_{3}$ or $S_{21} \rightarrow S_{3}$ with the probabilities $p_{21}$ or $p_{23}$ such that $p_{21}+p_{23}=1$. The customers arriving at the rates $\lambda_{1}^{\prime}, \lambda_{2}^{\prime}$ and $\lambda_{3}^{\prime}$ follow the network of servers $S_{21} \rightarrow S_{3}$, $S_{22} \rightarrow S_{3}$ and $S_{23} \rightarrow S_{3}$. After coming out from the server $S_{3}$.i.e. the Phase I of service, customers/jobs has to be processed through a system of equipments/machines (Phase II of service) taken on rent.

The following notations have been used in processing of jobs through the system of equipments/machines:

$S \quad$ : Sequence of jobs obtained by applying Johnson's procedure

$M_{j} \quad$ : Machine $j, j=1,2,3 \ldots \mathrm{m}$

$A_{i, j} \quad$ : Processing time of $i^{\text {th }}$ job on machine $M_{j}$

$L_{j}(S) \quad$ : The latest time when machine $M_{j}$ is taken on rent for sequence $S$

$t_{i, j}(S) \quad$ : Completion time of $i^{\text {th }}$ job for sequence $S$ on machine $M_{j}$

$t_{i, j}^{\prime}(S)$ : Completion time of $i^{\text {th }}$ job for sequence $S$ on $M_{j}$ when $M_{j}$ start processing jobs at time $L_{j}(S)$ 
$I_{i, j}(S) \quad$ : Idle time of machine $M_{j}$ for job $i$ in the sequence $S$

$U_{j}(S) \quad$ : Utilization time for machine $M_{j}$

$R(S) \quad$ : Total minimum rental cost of machines for the sequence $S$ of jobs

$C_{j} \quad:$ Rental cost for machine $M_{j}(j=1,2,3 \ldots m)$ per unit of time.

Let $P_{n_{1}, n_{2}, n_{3}, n_{4}, n_{5}, n_{6}}$ be the joint probability that there are $n_{l}$ units waiting in queue $Q_{l}$ in front of $S_{l 1}, n_{2}$ units waiting in queue $Q_{2}$ in front of $S_{12}, n_{3}$ units waiting in queue $Q_{3}$ in front of $S_{21}, n_{4}$ units waiting in queue $Q_{4}$ in front of $S_{22}, n_{5}$ units waiting in queue $Q_{5}$ in front of $S_{23}$ and $n_{6}$ units waiting in queue $Q_{6}$ in front of $S_{3}$. Now proceeding on the lines of Gupta et al. (2011), the joint probability function for the proposed queue network (Phase I of service) is $P_{n_{1}, n_{2}, n_{3}, n_{4}, n_{5}, n_{6}}=\rho_{1}^{n_{1}} \rho_{2}^{n_{2}} \rho_{3}^{n_{3}} \rho_{4}^{n_{4}} \rho_{5}^{n_{5}} \rho_{6}^{n_{6}}\left(1-\rho_{1}\right)\left(1-\rho_{2}\right)\left(1-\rho_{3}\right)\left(1-\rho_{4}\right)\left(1-\rho_{5}\right)\left(1-\rho_{6}\right)$

Here, $\rho_{1}=\frac{\left(\lambda_{1}+\lambda_{2} p_{21}\right)}{\mu_{1}\left(1-p_{12} p_{21}\right)}, \rho_{2}=\frac{\left(\lambda_{2}+\lambda_{1} p_{12}\right)}{\mu_{2}\left(1-p_{12} p_{21}\right)}, \rho_{3}=\frac{\lambda_{1}^{\prime}}{\mu_{1}^{\prime}}, \rho_{4}=\frac{\lambda_{2}^{\prime}}{\mu_{2}^{\prime}} \rho_{5}=\frac{\lambda_{3}^{\prime}}{\mu_{3}^{\prime}}$ and

$\rho_{6}=\left[\frac{\lambda_{1}^{\prime}+\lambda_{2}^{\prime}+\lambda_{3}^{\prime}}{\mu_{3}}+\frac{p_{13}\left(\lambda_{1}+\lambda_{2} p_{21}\right)+p_{23}\left(\lambda_{2}+\lambda_{1} p_{12}\right)}{\mu_{3}\left(1-p_{12} p_{21}\right)}\right]$.

Also, the mean queue length (average number of customers/jobs) $=\mathrm{L}=\mathrm{L}_{1}+\mathrm{L}_{2}+\mathrm{L}_{3}+\mathrm{L}_{4}+\mathrm{L}_{5}+\mathrm{L}_{6}$

Where, $L_{1}=\frac{\rho_{1}}{1-\rho_{1}} \quad L_{2}=\frac{\rho_{2}}{1-\rho_{2}}, L_{3}=\frac{\rho_{3}}{1-\rho_{3}}, L_{4}=\frac{\rho_{4}}{1-\rho_{4}}, L_{5}=\frac{\rho_{5}}{1-\rho_{5}}$ and $L_{6}=\frac{\rho_{6}}{1-\rho_{6}}$.

The average waiting time of customers/jobs as given by Little's Law is $W=L / \lambda$.

\subsection{Definition}

Completion time of $i^{\text {th }}$ job on $j^{\text {th }}$ machine is defined as:

$$
t_{i, j}=\max \left(t_{i-1, j}, t_{i, j-1}\right)+A_{i . j} \text { for } j \geq 2 \text {. Also, } t_{i, j}=\max \left(t_{i, j-1}^{\prime}, t_{i-1, j}^{\prime}\right)+A_{i, j} \text {. }
$$

Completion time of $i^{\text {th }}$ job on $j^{\text {th }}$ machine when $M_{j}$ starts processing jobs at the latest time $L_{j}$ is denoted by $t_{i, j}^{\prime}$ and is defined as, $t_{i, j}^{\prime}=L_{j}+\sum_{k=1}^{i} A_{k, j}=\sum_{k=1}^{i} I_{k, j}+\sum_{k=1}^{i} A_{k, j}$. Also, $t_{i, j}^{\prime}=\max \left(t_{i, j-1}, t_{i-1, j}^{\prime}\right)+A_{i, j}$

Further, the machines will be taken on rent as and when they are required and will be returned as and when they are no longer required. i.e. the first machine will be taken on rent in the starting of the processing the jobs, the second machine will be taken on rent at time when the first job is completed on the first machine and so on continuing in this way, the $m^{\text {th }}$ machine will be taken on rent when the first job is completed on the $(m-1)^{\text {th }}$ machine.

3.2. Theorem: Let ' $n$ ' jobs $J_{1}, J_{2}, J_{3} \ldots J_{n}$ are processed through ' $m$ ' machines $M_{j}(j=1,2 \ldots \mathrm{m})$ in order $M_{1}-M_{2}-M_{3}-\ldots \ldots . M_{m}$ with no passing allowed. If $A_{i, j}$ represents the processing time of $i^{\text {th }}$ job $(i=1$, $2, \ldots, \mathrm{n})$ on $\boldsymbol{j}^{\text {th }}$ machine $(\boldsymbol{j}=\mathbf{1}, \mathbf{2}, \ldots ., \mathrm{m})$ such that $\min A_{i, s} \geq \max A_{i,(s+1)} ; \boldsymbol{s}=1,2, \ldots,(\boldsymbol{m}-2)$, then the optimal schedule minimizing the total elapsed time is given by the following decision rule: Job $J_{k}$ proceeds job $\boldsymbol{J}_{\boldsymbol{k}+1}$ if $\min \left\{G_{k}, H_{k+1}\right\}<\min \left\{G_{k+1}, H_{k}\right\}$; Where, $G_{i}=A_{i, 1}+A_{i, 2}+---+A_{i,(m-1)}$ and $H_{i}=A_{i, 2}+A_{i, 3}+---+A_{i, m}$.

3.3. Theorem: The processing of the jobs on $\boldsymbol{m}^{\text {th }}$ machine $M_{\boldsymbol{m}}$ at the latest time $L_{\boldsymbol{m}}=\sum_{i=1}^{n} I_{i, m}$ keeps the total elapsed time $t_{n, m}$ remain unaltered.

3.4. Theorem: The processing of jobs on $\boldsymbol{j}^{\text {th }}$ machine $M_{j}$ at the latest time $L_{j}=\min _{1 \leq k \leq n}\left\{Y_{k j}\right\}$ keeps the total elapsed time $t_{n, m}$ remain unaltered, where $Y_{1 j}=L_{j+1}-A_{1, j}$ and $Y_{k j}=t_{(k-1),(j+1)}^{\prime}-\sum_{i=1}^{k} A_{i, j} ; k>1 ; j=(m-1),(m-2), \ldots . ., 3,2$.

\section{PROPOSED ALGORITHM}

The following algorithm is proposed to find the optimal sequence of the jobs processing with minimum makespan, the latest time at which equipments/machines should be taken on rent, minimum mean queue 
length of jobs processing, when the completion time (waiting time + service time) of the jobs in getting phase I of service will be the setup time for first machine $M_{l}$ in phase II of service.

Step 1: Find the mean queue length on the lines of Gupta et al. (2011) using the formula

$$
L=\frac{\rho_{1}}{1-\rho_{1}}+\frac{\rho_{2}}{1-\rho_{2}}+\frac{\rho_{3}}{1-\rho_{3}}+\frac{\rho_{4}}{1-\rho_{4}}+\frac{\rho_{5}}{1-\rho_{5}}+\frac{\rho_{6}}{1-\rho_{6}} .
$$

Step 2: Find the average waiting time of the customers on the line of Little's (1965) using relation $E(w)=\frac{L}{\lambda}$, where $\lambda=\lambda_{1}+\lambda_{2}+\lambda_{1}^{\prime}+\lambda_{2}^{\prime}+\lambda_{3}^{\prime}$.

Step 3: Find the completion time $(C)$ of jobs/customers coming out of Phase I .i.e. when processed through the network of queues (Phase I of service) by using the formula

$$
C=E(W)+\frac{1}{\mu_{1} p_{12}+\mu_{1} p_{13}+\mu_{2} p_{21}+\mu_{2} p_{23}+\mu_{3}+\mu_{1}^{\prime}+\mu_{2}^{\prime}+\mu_{3}^{\prime}} .
$$

Step 4: The completion time C of the customers / jobs through the network of queues (Phase I of service) will be the setup time for machine $M_{1}$ in Phase II of service. Define machines $M_{1}, M_{2}, M_{3}$------ $M_{m}$ with processing time $A_{i 1}^{\prime}=A_{i 1}+C$ and $\dot{A_{i, j}}=A_{i, j} ; \quad i=1,2,3, \ldots, n ; j=2,3, \ldots, m$.

Step 5: Check the structural condition: $\operatorname{Min} A_{i, s}^{\prime} \geq \operatorname{Max} A_{i,(s+1)} ; s=1,2,3,4 \ldots \ldots \ldots$

If these conditions are satisfied then go to Step 6, else the data is out of scope of present algorithm.

Step 6: Introduce two fictitious machines $G$ and $H$ with processing times $G_{i}$ and $H_{i}$ defined as $G_{i}=\dot{A}_{i, 1}+\dot{A_{i, 2}}+\dot{A_{i, 3}}+\ldots \ldots .+\dot{A}_{i,(m-1)}$ and $H_{i}=\dot{A_{i, 2}}+\dot{A}_{i, 3}+\dot{A}_{i, 4}+\ldots \ldots .+A_{i, m}^{\prime}$ for all $i .$.

Step 7: Using Johnson's procedure, obtain the sequence $\{S\}$ of jobs with minimum total elapsed time and calculate total elapsed time $t_{n, p}(S) ; p=2,3,4 \ldots m$.

Step 8: Find the latest time $L_{m}$ of $m^{t h}$ machine $M_{m}$ for sequence $\{S\}$ of jobs as $L_{m}(S)=t_{n, m}(S)-\sum_{i=1}^{n} A_{i, m}$.

Step 9: For the sequence $\{S\}$ of jobs, calculate the latest time of machines $M_{j}(j=2,3, \ldots, m-1)$ and their utilization time as follows:

$$
\begin{aligned}
& Y_{1 m}(S)=L_{m}(S)-A_{1,(m-1)}(S), Y_{q m}(S)=L_{m}(S)+\sum_{i=1}^{q-1} A_{i, m}(S)-\sum_{i=1}^{q} A_{1,(m-1)}(S) ; q=2,3, \ldots \ldots, n \\
& L_{m-1}(S)=\min _{1 \leq q \leq n}\left\{Y_{q m}(S)\right\}, U_{m-1}(S)=t_{n(m-1)}(S)-L_{m-1}(S) .
\end{aligned}
$$

Now, for $j=(m-1),(m-2) \ldots . . .3$; calculate

$$
\begin{aligned}
& Y_{1 j}(S)=L_{j}(S)-A_{1,(j-1)}(S), Y_{q j}(S)=L_{j}(S)+\sum_{i=1}^{q-1} A_{i, j}(S)-\sum_{i=1}^{q} A_{1,(j-1)}(S) ; q=2,3, \ldots \ldots, n \\
& L_{(j-1)}(S)=\min _{1 \leq q \leq n}\left\{Y_{q j}(S)\right\} \text { and } U_{(j-1)}(S)=t_{n(j-1)}(S)-L_{(j-1)}(S) .
\end{aligned}
$$

Step 10: Find the total minimum rental cost of the machines for the optimal sequence $\{S\}$ of jobs using the relation $R(S)=\sum_{i=1}^{n} A_{i 1} \times C_{1}+U_{2}(S) \times C_{2}+U_{3}(S) \times C_{3}+----+U_{m-1}(S) \times C_{m-1}+\sum_{i=1}^{n} A_{i m} \times C_{m}$.

The sequence $\{S\}$ will be the optimal sequence of jobs processing with minimum total elapsed time $t_{n, m}$, minimum rental cost of the equipments/machines $R(S)$ under the restrictive hiring policy, with mean queue length $\mathrm{L}$ and average waiting time $\mathrm{E}(\mathrm{w})$.

\section{TEST OF PROPOSED ALGORITHM}

Consider fourteen customers / jobs are to be processed through the network of queues with the servers $S_{1}$, $S_{2}$ and $S_{3}$, Server $\mathrm{S}_{3}$ is commonly linked in series with each of two servers $\mathrm{S}_{1}$ and $\mathrm{S}_{2}$. The number of the customers, mean arrival rate, mean service rate and associated probabilities are as given in table 1. 
Sharma, S. et al., Analysis of Queuing Scheduling Linkage Model to Minimize the Hiring Cost....

Table 1: The detail classification of the linkage model

\begin{tabular}{|l|l|c|l|l|}
\hline S. No. & No. of Customers & Mean Arrival Rate & Mean Service Rate & Probabilities \\
\hline 1 & $\mathrm{n}_{1}=4$ & $\lambda_{1}=3$ & $\mu_{1}=10$ & $\mathrm{p}_{12}=0.4$ \\
\hline 2 & $\mathrm{n}_{2}=2$ & $\lambda_{2}=5$ & $\mu_{2}=9$ & $\mathrm{p}_{13}=0.6$ \\
\hline 3 & $\mathrm{n}_{3}=3$ & $\lambda_{1}^{\prime}=2$ & $\mu_{1}^{\prime}=7$ & $\mathrm{p}_{21}=0.6$ \\
\hline 4 & $\mathrm{n}_{4}=2$ & $\lambda_{2}^{\prime}=4$ & $\mu_{2}^{\prime}=8$ & $\mathrm{p}_{23}=0.4$ \\
\hline 5 & $\mathrm{n}_{5}=3$ & $\lambda_{3}^{\prime}=5$ & $\mu_{3}^{\prime}=9$ & \\
\hline 6 & $\mathrm{n}_{6}=14$ & & $\mu_{3}=22$ & \\
\hline
\end{tabular}

After getting Phase I of service, jobs/customers are to be processed on equipments/machines $M_{1}, M_{2}, M_{3}$ and $M_{4}$ with processing time $A_{\mathrm{i}, 1}, A_{\mathrm{i}, 2}, A_{\mathrm{i}, 3}$ and $A_{\mathrm{i}, 4}$ respectively as given in table 2 .

Table 2: The machines $M_{1}, M_{2}, M_{3}$ and $M_{4}$ with processing times

\begin{tabular}{|l|l|l|l|l|l|l|l|l|l|l|l|l|l|l|}
\hline Jobs & 1 & 2 & 3 & 4 & 5 & 6 & 7 & 8 & 9 & 10 & 11 & 12 & 13 & 14 \\
\hline$M_{I}\left(A_{i, 1}\right)$ & 15 & 20 & 18 & 17 & 16 & 19 & 17 & 20 & 15 & 16 & 17 & 19 & 18 & 15 \\
\hline$M_{2}\left(A_{i, 2}\right)$ & 14 & 13 & 12 & 14 & 10 & 11 & 12 & 13 & 11 & 12 & 13 & 14 & 10 & 12 \\
\hline$M_{3}\left(A_{i, 3}\right)$ & 9 & 8 & 9 & 10 & 7 & 8 & 10 & 9 & 7 & 8 & 10 & 7 & 8 & 9 \\
\hline$M_{4}\left(A_{i, 4}\right)$ & 6 & 5 & 3 & 5 & 4 & 3 & 6 & 5 & 4 & 5 & 3 & 2 & 6 & 4 \\
\hline
\end{tabular}

The objective is to find an optimal sequence of the jobs / customers processing to minimize the makespan and rental cost of equipments/machines for the proposed queue-scheduling linkage model by considering the first phase service if the rental cost per unit of time for the machines $M_{1}, M_{2}, M_{3}$ and $M_{4}$ are 8, 10, 12 and 6 per unit of time.

Here, we observe that

$$
\rho_{1}=0.789474, \rho_{2}=0.96433, \rho_{3}=0.285714, \rho_{4}=0.5, \rho_{5}=0.555556, \rho_{6}=0.668262 \text {. }
$$

The mean queue length $=$ Average number of jobs $/$ customers $=L=18.101925$ units, and Average waiting time of the jobs / customers $=0.952733$ units.

The total completion time of jobs/customers when processed through network of queues $C=0.96811$ units.

On taking this completion time as the setup time for the first machine $M_{1}$ in Phase II of service and on using various steps of the proposed algorithm, we obtain $S=4-1-7-8-2-11-14-10-13-3-12-9-6-$ 5 as an optimal sequence of jobs processing with minimum total elapsed time as 276.553619 units.

The latest times at which machine $M_{4}, M_{3}$ and $M_{2}$ should be taken on rent are $L_{4}=215.553619$ units, $L_{3}=153.553619$ units, and $L_{2}=94.553619$ units.

The utilization time of machines $M_{2}$ and $M_{3}$ are $U_{2}(S)=171, U_{3}(S)=119$ units and the minimum possible rental cost of machines will be $\mathrm{R}(S)=5200.428711$ units.

\section{CONCLUSION AND DISCUSSION}

In this paper an algorithm is developed to minimize the hiring cost of equipments/machines taken on rent under a restrictive hiring policy for the proposed queuing scheduling linkage model. The latest times at which various equipments/machines must be hired so as to minimize the utilization time and hence their hiring/rental cost with minimum makespan, average waiting time and with minimum mean queue length are derived. Using Johnson's technique, the minimum makespan for the above discussed numerical illustration comes to be 276.553619 units with total possible hiring cost of equipments/machines taken on rent is 5538.5612 units. If the proposed algorithm is applied, the minimum makespan comes to be same and the total possible hiring cost of equipments/machines taken on rent under the same hiring policy come to be 5200.428711 units, which is comparatively less than the hiring cost as calculated by using Johnson's technique. Hence, the proposed algorithm is more efficient as it optimize both the criteria's simultaneously for a given phase I of the service. The study may further be extended by introducing the concept of fuzziness 
Sharma, S. et al., Analysis of Queuing Scheduling Linkage Model to Minimize the Hiring Cost....

in processing of jobs/customers and by including some more constraints in the linkage model such as independent setup time, transportation time and weightage of jobs etc.

\section{REFRENCES}

Bagga, P.C.(1968). Sequencing in a rental situations. Journal of Canadian Operation Research Society, 7, $152-153$

Bagga, P.C., Bhambani, A. (2000). Bicriteria in a two-stage flowshop scheduling problem. Indian J. pure appl. Math., 31(11): 1475-1481.

Erlang, A.K. (1909). The theory of probabilities and telephone conversations. Nyt Tidsskrift fur Matematik, B (20), 33-63.

Gupta, D., Sharma, S. and Gulati, N. (2011). On steady state behaviour of a network queuing model with biserial and parallel channels linked with a common server. Computer Engineering and Intelligent systems, 2(3), 11-22.

Gupta, D. and Sharma, S. (2011). Minimizing rental cost under specified rental policy in two, processing time associated with probabilities including break-down interval and job-block criteria. European Journal of Business and Management, 3(2), 268-286.

Gupta, D., Sharma, S. and Gulati, N. (2011). Bicriteria in n x 3 flowshop scheduling under specified rental policy, processing time associated with probabilities including job block criteria. Proceeding of International Conference in AMOC-2011, I.I.T., Roorkee, 398-407.

Gupta, D., Sharma, S. and Seema (2012a). On linkage of a flowshop scheduling model including job block criteria with a parallel biserial queue network. Computer Engineering and Intelligent System, 3(2), 17-28.

Gupta, D., Sharma, S. and Seema (2012b). On linkage of a queue network with biserial and parallel channels linked with a common server to a two stage flowshop scheduling system. International Journal of Emerging Trends in Engineering and Development, 2(2), 265-275.

Gupta, D., Sharma, S. and Seema (2012c). On linkage of parallel biserial server linked with a common server to a three stage flowshop scheduling model. International Journal of Applied Physics and Mathematics, 2(3), pp. 152-155.

Gupta, D., Sharma, S. and Seema (2012d). A mathematical model for linkage of a queuing network with a flowshop scheduling system. International Journal of Mathematical Sciences, 11(3-4), 197-213.

Gupta, D., Sharma, S. and Seema (2013). A mathematical analysis on linkage of network of queue with two machines in a flowshop including transportation time. International J. of applied Operational Research, 2,(4), 1-18.

Jackson, R. R. P. (1954). Queuing system with phase type service. Operation Research Quarterly, 5, 109120.

John, D. C. (1965). A proof of queuing formula: “ $L=\lambda W "$. Operation Research, 13, 400-412.

Johnson, S. M. (1954). Optimal two \& three stage production schedules with set up times includes. Nav. Res. Log. Quart.. 1, 61-68.

Kumar, V., Singh, T. P. and Kumar, R. (2007). Steady state behaviour of a queue model comprised of two subsystems with biserial linked with common channel. Reflection des ERA, 1(2), 135-152.

Maggu, P. L. (1970). Phase type service queue with two channels in Biserial. Journal of Operational Research Society of Japan, 13(1), 1-6.

Maggu, P. L. and Gupta, S. (2007). On a network of two queues in bitandem linked in series with a network of two machines in a flowshop. J. Indian Soc. Stat. Opers. Res., XXVIII (1-4), 1-9.

Singh, T. P., Kumar, V. and Kumar, R. (2005). On transient behaviour of a queuing network with parallel biserial queues. JMASS, 1(2), 68-75.

Smith, W.E. (1956). Various optimizers for single stage production. Naval Research Logistics, 3:59-66.

Singh, T. P., Kumar, V. (2009). On linkage of a scheduling system with biserial queue network. Arya Bhatta Journal of Mathematics \& Informatics, 1(1), 71- 76. 